\title{
El estudiante de TSU en gastronomía ante el inicio de la pandemia, percepción y adaptación a una modalidad virtual
}

\section{The UST student in gastronomy before the start of the pandemic, perception and adaptation to a virtual modality}

\author{
GARCÍA-RAMÍREZ, Karina Nayeli †* \\ Universidad Tecnológica de León, Blvd. Universidad Tecnológica \#225 Col. San Carlos CP37670 León, Gto. México.
}

ID $1^{\mathrm{er}}$ Autor: Karina Nayeli, García-Ramirez

DOI: $10.35429 / J U P .2020 .12 .4 .20 .27$

Recibido: 20 de Julio, 2020; Aceptado 30 de Diciembre, 2020

\begin{abstract}
Resumen
La inminente llegada a México de la pandemia por COVID-19 trastocó a propios y extraños que nunca imaginaron las consecuencias de este hecho atroz que modificó la dinámica de la sociedad mundial. En el sector educativo el cambio radical a una modalidad virtual dejó en evidencia la gran brecha digital que existe y en el estudiantado de TSU en Gastronomía de la UTL, este cambió significó repensar sobre el desarrollo de las competencias propias de la carrera, así como de las habilidades técnicas y culinarias obligadas de cada materia. Sin embargo, pronto el hecho quedó de lado al comprender que esta adaptación exigiría mucho más que el sólo poder cocinar en un laboratorio especializado, se trataba de aprender a administrar los tiempos, a comunicarse asertivamente, a convivir en familia y a retroalimentar a través de un monitor las enseñanzas de los docentes, quiénes también enfrentaban el reto de compartir conocimientos frente a una pantalla.
\end{abstract}

Estudiantes, Percepción, Aprendizaje

\begin{abstract}
The imminent arrival in Mexico of the COVID-19 pandemic upset locals and strangers who never imagined the consequences of this atrocious event that modified the dynamics of world society. In the education sector, the radical change to a virtual modality revealed the great digital gap that exists and in the TSU student body in Gastronomy of the UTL, this change meant rethinking about the development of the competences of the career, as well as the technical and culinary skills required of each subject. However, the fact was soon put aside when understanding that this adaptation would require much more than just being able to cook in a specialized laboratory, it was about learning to manage time, to communicate assertively, to live together as a family and to provide feedback through a monitor the teachings of teachers, who also faced the challenge of sharing knowledge in front of a screen.
\end{abstract}

Students, Perception, Learning

Citación: GARCÍA-RAMÍREZ, Karina Nayeli. El estudiante de TSU en gastronomía ante el inicio de la pandemia, percepción y adaptación a una modalidad virtual. Revista de Políticas Universitarias. 2020. 4-12:20-27.

\footnotetext{
* Correspondencia al Autor (Correo electrónico: nramirez@utleon.edu.mx)

$\dagger$ Investigador contribuyendo como primer autor.
} 


\section{Introducción}

El año 2020 ha establecido un reto en el proceso de enseñanza-aprendizaje, de un día para otro los edificios fueron cerrados y la formación del estudiante fue modificada a un entorno $100 \%$ virtual. Sin tiempo de preparación, ni análisis de los recursos y las posibilidades.

La pandemia de COVID-19 derivó en el confinamiento de prácticamente todos los ciudadanos del mundo; un sector importante de este grupo es la comunidad universitaria: profesionistas en formación, ávidos de aprender $\mathrm{y}$ poner en práctica sus conocimientos $\mathrm{y}$ docentes de frente a un monitor, en un intento interminable de captar la atención y generar un aprendizaje significativo. Transformando a las nuevas tecnologías en aliadas para la continuidad de la educación, ahora a distancia. "Al tiempo que se cerraban las puertas de las universidades, se abrían las puertas de las tecnologías". (Grynspan, 2020)

Sumando el desafío de la brecha digital que existe, familias que con gran esfuerzo apenas han logrado la integración de sus hijos/as a la formación profesional. En el encuentro virtual de expertos en la formación superior: "Diálogo sobre el impacto de la pandemia en la educación superior y la transformación digital de las universidades en Iberoamérica" se mencionaron estos y otros retos que desde marzo 2020 obligatoriamente han estado presentes en este sector, sin embargo, el cambio y adaptación a las tecnologías digitales es algo que ya se trabajaba en la última década.

En la carrera de TSU en Gastronomía esta incertidumbre impactó en el desarrollo de las clases prácticas, alumnado habituado a poner en práctica sus conocimientos y destrezas dentro de una cocina/laboratorio bajo el ojo visor y guía del chef/docente; ahora debía apostar a una pausa sin fecha límite y pronto buscar alternativas para el desarrollo de estas habilidades con los insumos y materiales que se tienen al alcance, con una supervisión en línea, pocas veces igual de eficiente y con recursos propios que implicaron gastos extras.
Aprender a grabar, tomar imágenes de calidad, edición de videos, desenvolverse a través de una cámara, son las "nuevas habilidades" aprendidas prácticamente por la necesidad y que pueden llegar a sobrepasar la carga emocional del estudiantado, llevándonos ahora al tema de garantizar la integridad de cada persona, docentes y estudiantes, pues la UTL tiene en su misión la formación integral del ciudadano del mundo. Bajo este contexto y con la experiencia del cambio, se formuló un cuestionario al alumnado de tercer cuatrimestre de la carrera de TSU en Gastronomía, enfocado, más que en sus habilidades culinarias, en su percepción del entorno y sus posibilidades tecnológicas, algo que permitiera no sólo evaluar la continuidad en el sistema educativo sino comprender los factores que directamente afectan sus propias decisiones y la necesidad de orientación y apoyo.

\section{Metodología}

Se realizó una encuesta a través de la plataforma Forms con el objetivo de conocer las percepciones del estudiantado de tercer cuatrimestre de la carrera de TSU en Gastronomía de la UTL ante la pandemia por COVID-19 y la educación en línea. Se diseñaron 9 cuestionamientos abiertos que reflejaran la situación familiar y escolar de cada encuestado. La carrera en el momento de la actividad se conformaba por tres grupos de tercero, y se programó para un grupo total de 30 estudiantes, el $34 \%$ de la matrícula general, obteniendo una respuesta efectiva de sólo el $73 \%$, lo que ya mostraba un primer resultado de deserción principalmente por motivos económicos.

\section{Objetivos}

Reconocer las percepciones del estudiantado, sus posibilidades tecnológicas y económicas, así como el riesgo de deserción desencadenado por estos mismos motivos. 


\section{Resultados}

\section{1. ¿Qué equipo electrónico utilizas para} conectarte a clases?

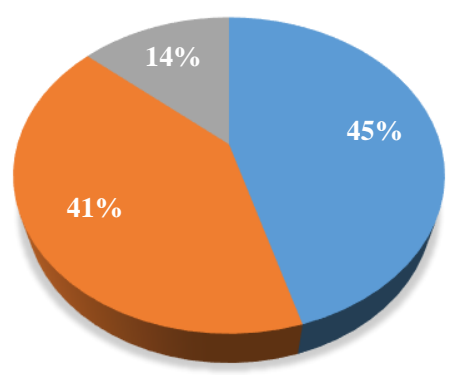

nComputadora/Laptop $\square$ Celular $\square$ Ambos

Gráfico 1 ¿Qué equipo electrónico utilizas para conectarte a clases?

En el resultado global se muestra que todo el estudiantado cuenta con un dispositivo que le permite la conexión, lo que los sitúa en un buen estándar de equidad. Como se sabe, actualmente la niñez y la juventud tienen mayores posibilidades de acceso a los dispositivos móviles. De acuerdo con el INEGI, estudio 2019, en México el $43.0 \%$ de Usuarios de computadora como proporción de la población de seis años o más de edad. Mientras que el $44.6 \%$ de Usuarios de computadora que la usan como herramienta de apoyo escolar.

En nuestra población de la carrera encontramos que un $45 \%$ cuenta con una computadora o laptop, el $44 \%$ casi la misma cantidad, tiene un celular y un $14 \%$ cuenta con ambos dispositivos. Un dato curioso es que ninguno menciona el uso de Tabletas y sólo 1 estudiante dijo tener una computadora de escritorio.

\section{El servicio de internet que uso es:}

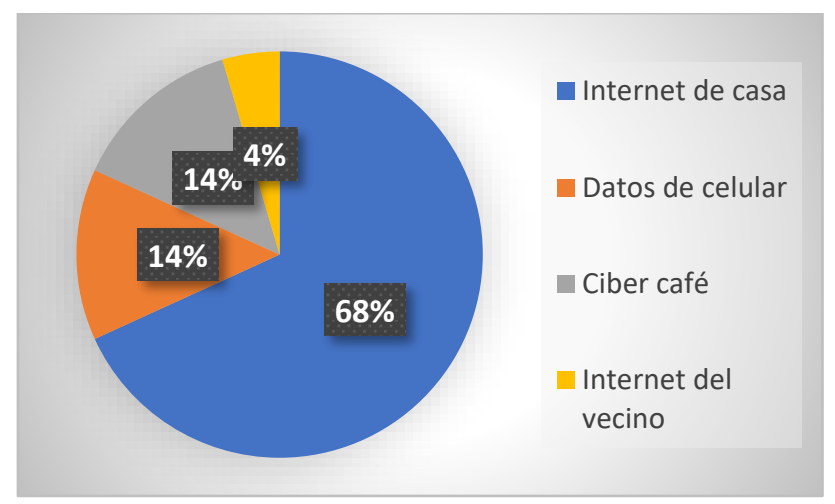

Gráfico 2 El servicio de internet que uso es:
Al respecto de la conectividad, el servicio de internet se contempla que más de la mitad $(68 \%)$ cuentan con servicio de internet en casa. Una cantidad igual del $14 \%$ hace uso de los datos en el celular y/o asiste a un ciber café, mientras que el mínimo de ellos (4\%) hace uso de la red contratada por un vecino. Bajo estas circunstancias lo destacable es el esfuerzo e interés de las y los estudiantes por mantenerse conectados para asistir a sus clases y cumplir con sus obligaciones escolares.

\section{3. ¿Cómo describes tu experiencia escolar en estos meses de confinamiento?}

\begin{tabular}{l}
$\begin{array}{l}\text { Mala, la verdad a veces la comunicación no es tan } \\
\text { interactiva. No sé aprende lo suficiente y la carrera no se } \\
\text { presta a qué sea en línea }\end{array}$ \\
\hline No muy buena \\
\hline Realmente no sé toma la atención necesaria \\
\hline A sido complicada \\
\hline $\begin{array}{l}\text { En lo personal no me gusta estar detrás de una pantalla, } \\
\text { por qué no es lo mismo que estar presente }\end{array}$ \\
\hline $\begin{array}{l}\text { Un poco estresante ya que las dudas que surgían eran } \\
\text { atendidas en un buen rato, no se aprende igual que en las } \\
\text { clases presenciales } \\
\text { La participación es menor y la motivación igual. } \\
\text { Aparte la práctica es muy necesaria y más en la carrera }\end{array}$ \\
\hline Deficiente \\
\hline $\begin{array}{l}\text { La verdad es difícil, por suerte era el último cuatri y eran } \\
\text { estadías porque si hubieran sido clases normales la } \\
\text { verdad no hubiera aprendido nada. }\end{array}$ \\
\hline $\begin{array}{l}\text { Una muy mala experiencia por parte de que los } \\
\text { profesores no tenían una buena actitud para aclarar } \\
\text { dudas }\end{array}$ \\
\hline $\begin{array}{l}\text { Más o menos puesto que en el otro cuatri a veces no } \\
\text { dormía por entregar los trabajos a tiempo. }\end{array}$ \\
\hline Buenos \\
\hline Buena, pero no tan buena como la presencial \\
\hline Un poco difícil la comprensión de temas \\
\hline Regular \\
\hline algo complicada por qué no cuento con internet \\
\hline $\begin{array}{l}\text { Pues un poco difíciles ya que había veces que se } \\
\text { cortaban el video llamadas y no era tan fácil entender las } \\
\text { clases }\end{array}$ \\
\hline $\begin{array}{l}\text { Fue buena, aprendí a manejar mejor las tecnologías y } \\
\text { herramientas para implementar mis conocimientos. }\end{array}$ \\
\hline $\begin{array}{l}\text { La verdad no he aprendido mucho porque los maestros } \\
\text { no siempre nos daban clase porque tienen más grupos, } \\
\text { aun así, solo duraban } 1 \text { hora. }\end{array}$ \\
\hline $\begin{array}{l}\text { Es mucho más práctico, pero no hay nada como poder } \\
\text { interactuar con tu profesor y hacerle saber tus } \\
\text { inquietudes y dudas. }\end{array}$ \\
\hline $\begin{array}{l}\text { Es bueno, pero lo malo es que las clases en línea no son } \\
\text { indicadas para responder dudas }\end{array}$ \\
\hline $\begin{array}{l}\text { Una experiencia tranquila e interesante al descubrir } \\
\text { nuevas plataformas tecnológicas para dar seguimiento a } \\
\text { las clases. }\end{array}$ \\
\hline Mala experiencia \\
\hline
\end{tabular}

Gráfico 3 ¿Cómo describes tu experiencia escolar en estos meses de confinamiento? 
La dificultad ante la nueva modalidad se percibe en las respuestas de las y los estudiantes, quiénes han enfrentado como un reto adaptarse a estar detrás de un monitor, luego de una formación académica completamente presencial desde el inicio de su escolaridad, la interacción a través de la pantalla con el docente se precisa impersonal e impacta en el interés y la atención. Varios de los casos han destacado que en casa no se tiene un espacio libre y único para tomar clases y que la dinámica de la familia llega a irrumpir en el sentido de la clase, haciéndose aún más difícil el aprendizaje. Como docente, estos argumentos son valiosos, ya que también se enfrenta el desánimo y la construcción constante de materiales interactivos que provean dinámica a las sesiones y motiven al estudiantado. Así como buscar espacios virtuales de atención personalizada a la necesidad de cada uno/a.

\section{4. ¿Qué has aprendido durante estos meses de cuarentena?}

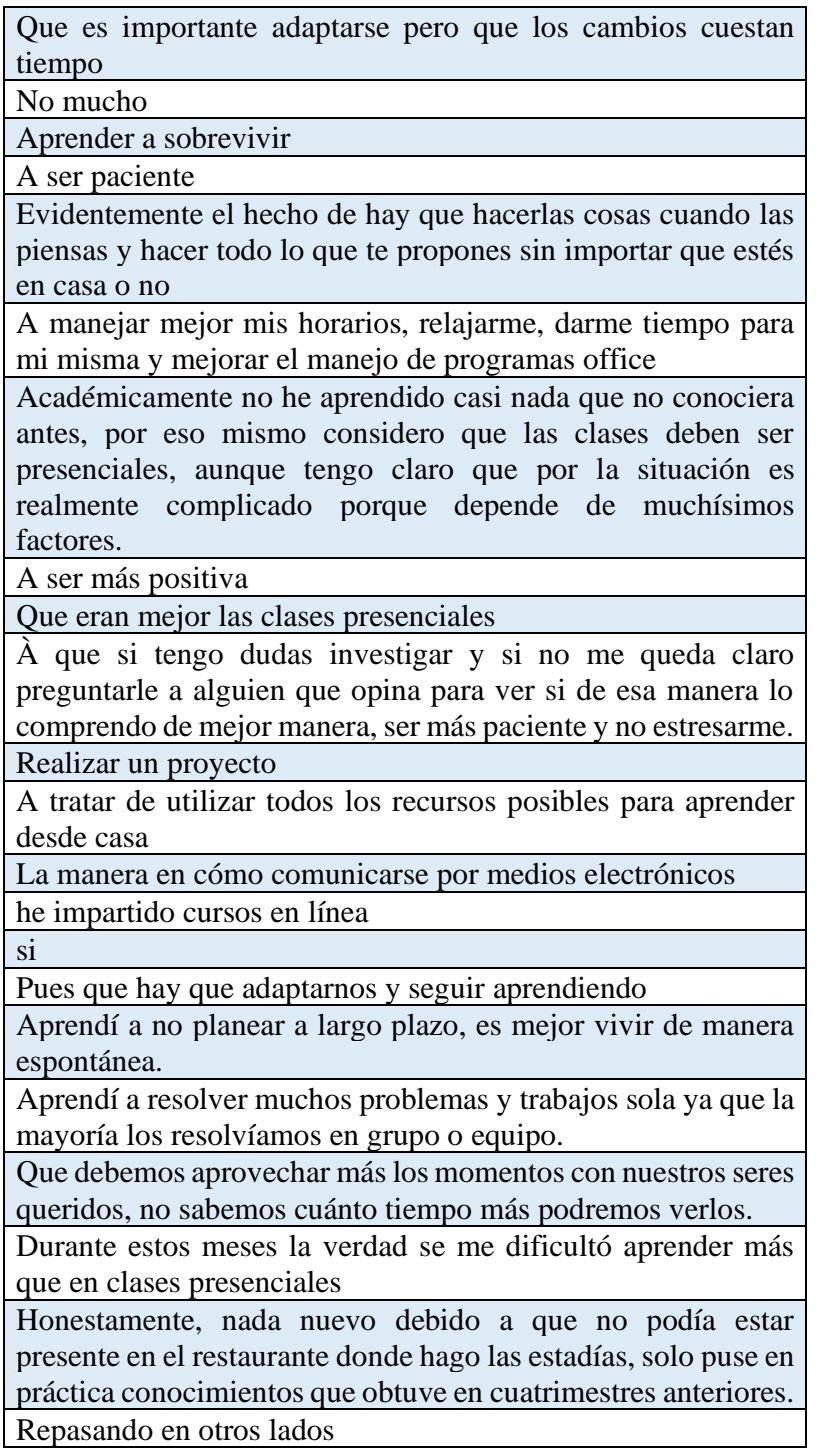

Gráfico 4 ¿Qué has aprendido durante estos meses de cuarentena?
El confinamiento, sin embargo, ha dejado grandes aprendizajes en el manejo de las emociones y la administración del tiempo. La evidencia nos deja claro que la juventud tiene gran capacidad de adaptación y también gran interés por seguir sus estudios y continuar aprendiendo. La pandemia como un momento difícil que enfrenta la sociedad nos lleva también a valorar a los seres que están a nuestro alrededor y que acompañan a nuestro alumnado en esta etapa. Convivir y compartir espacios, adecuar horarios, vivir emociones de presión y desánimo pueden conjugarse en el aprovechamiento de estas circunstancias para aprender y dar valor agregado a los apoyos recibidos. El acompañamiento que reciben nuestros/as estudiantes por parte del docente nos ha abierto las puertas de su hogar y acercarnos a su familia y a un día a día que se construye en conjunto.

\section{5. ¿Cómo se adaptó tu dinámica familiar a estos cambios, durante el confinamiento?}

Pues creo que aún no se adaptan un $100 \%$. A veces creen que no tengo escuela y me piden hacer otras cosas

En la forma de respetar la hora de clase o asesoría

Los roles no están bien ya que ahora buscamos trabajo

Fue muy adaptable

Han Sido unos días de peleas, pero sin embargo nos hemos apoyado en todo

Se informaba cuando había clase o reunión para evitar ruidos o interrupciones, aunque la mayoría de las veces son en la mañana

Simplemente quedarnos en casa y cada uno realizar sus actividades.

Ha estado difícil, mi papá de quedó sin trabajo y pues nadie más de mi casa trabaja, pero pues apoyamos con lo que podemos

Sin comunicación

Más o menos debido a que tenían que salir aun así de casa a trabajar.

Muy bien, solo hubo cambios relacionados con el deporte

Bien, aunque realmente si es pesado estar tanto tiempo en casa

Bien

regular

fue algo difícil, pero aumento la convivencia

Pues en hacer las labores de la casa con las clases

Nos adaptamos prácticamente a los cambios y reconocimos la importancia de la escuela en el proceso de aceptación en la familia.

Hubo un poco más de confianza para hablar y resolver los problemas, fue fácil ayudar en los que haceres porque estamos juntos.

Salir máximo una vez por semana por víveres y cuidar más de los más grandes y pequeños. Los que tienen que salir a trabajar, con las medidas de seguridad y sanidad necesarias al volver a casa.

En realidad, no cambiaba mucho, ya que siempre era la misma rutina, casa y escuela

Primero me concentraba en mis estudios, después ayudaba a las tareas del hogar.

Se adaptaron bien

Gráfico 5 ¿Cómo se adaptó tu dinámica familiar a estos cambios, durante el confinamiento?

GARCÍA-RAMÍREZ, Karina Nayeli. El estudiante de TSU en gastronomía ante el inicio de la pandemia, percepción y adaptación a una modalidad virtual. Revista de Políticas Universitarias. 2020 
Siguiendo en el tenor de encontrarnos ahora escuela y familia en el mismo espacio han hecho visualizar las responsabilidades de forma distinta, obligan a responder a todos los actores (familia, estudiante, docente) al cuidado mutuo y la sana convivencia, al respeto por los que trabajan, los que estudian, los que atienden a los demás. Ha sido una oportunidad valiosa de aumentar la armonía, de conocerse más a fondo, ampliar la comunicación. Las y los estudiantes se saben acompañados y respaldados por la institución y eso se llega a proyectar en el hogar como un resultado positivo de esta pandemia.

\section{6. ¿Consideras seguro para tu salud y la de tu comunidad volver a la escuela en los próximos meses?}

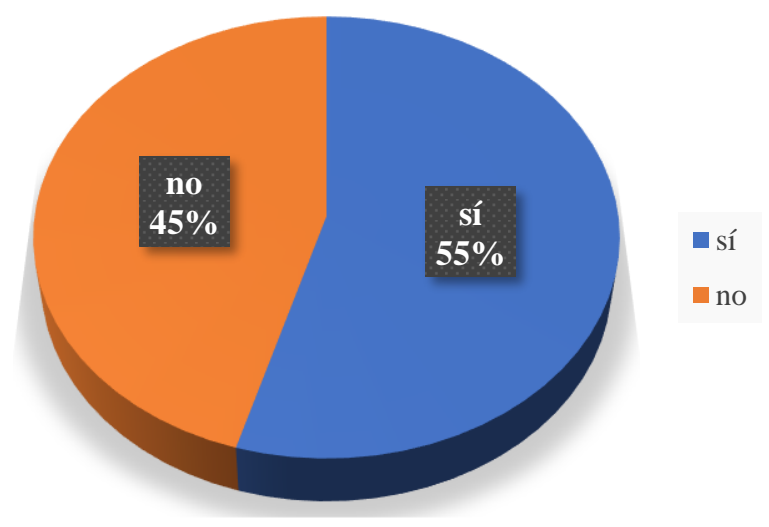

Gráfico 6 ¿Consideras seguro para tu salud y la de tu comunidad volver a la escuela en los próximos meses?

El contexto de este cuestionamiento es aquel que, en unos meses de cuarentena, vislumbraba la posibilidad de crear espacios seguros para un regreso controlado, al momento de la redacción de este artículo esta posibilidad es todavía lejana. No obstante, nos deja ver la necesidad del alumnado de retomar sus actividades "normales", motivados principalmente por ejecutar sus prácticas en los laboratorios adecuados y poder desarrollar sus habilidades en las técnicas culinarias que son el motor de su carrera. En el siguiente cuadro podremos reconocer la preocupación y consternación del comportamiento social que nos mantiene hoy en día en confinamiento, continuando con el análisis en el sector siguiente:

\section{1. ¿Por qué?}

Pienso que debemos adaptarnos a todo y seguir cuidando nuestra salud fortaleciendo nuestra cuerpo y sistema inmune nosotros mismos. Creo que sí debemos regresar con medidas de seguridad necesarias. La verdad es que no es sano quedarse en casa tanto tiempo, afecta la salud emocional y física y no es vivir, es intentar "sobrevivir". He ido constantemente al médico

Los casos han empeorado y hay menor trabajo por lo que el dinero para transportar no me ayudaría

Porque aún no termina la contingencia

Creo que tomando todas las medidas de higiene se puede Porque si así no hacen caso, llenado a las clases presenciales menos se va a controlar, los alumnos no tomarán cuidado sobre las medidas de higiene y porque es incómodo traer el cubre bocas todo el día, así que mejor en casita :)

Es difícil y estoy consciente que no es seguro, pero si muuuy necesario volver. Quizá Con todas las medidas necesarias, siguiendo protocolos de seguridad y esperando que en estas semanas la situación mejore considerando que somos personas responsables con nuestra salud y de los demás Tomando las medidas necesarias es posible ya que la verdad el tomar las clases desde la casa no es tan productivo

Necesitamos aprender algo

Eh leído que se han presentado personas con depresión debido a que no tienen con quien hablar, por otro lado, es mucho mejor las clases presenciales puesto que si tenemos una duda o algún inconveniente podemos ir directo y no esperar a que nos contesten, es mejor realizar prácticas y llevar a cabo las cosas en físico que por computadora investigar

Hay mucha gente irresponsable que sale, y al momento de regresar puede que haya más infectados

Creo que realmente se necesitaría que estén ya todos los casos recuperados es decir que todo esté en la normalidad, sin casos ni contagios por la salud y seguridad de los demás

Tomando precauciones y siguiendo los protocolos de seguridad ante todo para evitar problemas de salud.

es importante

hay muchos alumnos dentro de las instalaciones

Si vamos con las medidas necesaria si podemos volver a la escuela y así tener más conocimiento

Todas las personas tenemos conciencia de que debemos cuidarnos unos a otros, teniendo las medidas de higiene necesarias y correctas.

Porque no todos cumplen con lo que está sugiriendo el gobierno y no siguen las indicaciones adecuadas, aunque los alumnos necesitemos mejores explicaciones.

Por qué a estas alturas de la situación, hay muchas personas que siguen sin portar cubrebocas al salir, poniéndose en riesgo o peor aun poniendo en riesgo a las demás personas que por necesidad tienen que salir, y en su caso, poner en riesgo a sus familiares.

Ya somos lo bastante maduros para seguir protocolos de seguridad

Exposición constante al virus

Es mejor en el aula

Gráfico 7 Aunado a lo anterior ¿Por qué? 
El continuo debate entre la responsabilidad personal y la social, encamina a considerar muchas veces lo que uno mismo necesita o quiere antes del bien común. No es una mala postura, es el reflejo de la incertidumbre y la necesidad de convivir como seres sociales que somos. En definitiva, queda claro que el estudiantado no prevé la adaptación a un modelo $100 \%$ virtual en esta etapa de formación. Esta contingencia sigue percibiéndose pasajera y la exigencia a tomar las sesiones de cocina práctica en modalidad presencial es auténtica; las y los estudiantes no se perciben aprendiendo al máximo frente a un monitor. Esto sugiere mayor dinamismo del docente y reitera el reto de educar en el confinamiento.

\section{Expresa tus sugerencias para tus profesores en cuanto a la dinámica de clases virtuales y la mejora de estas.}

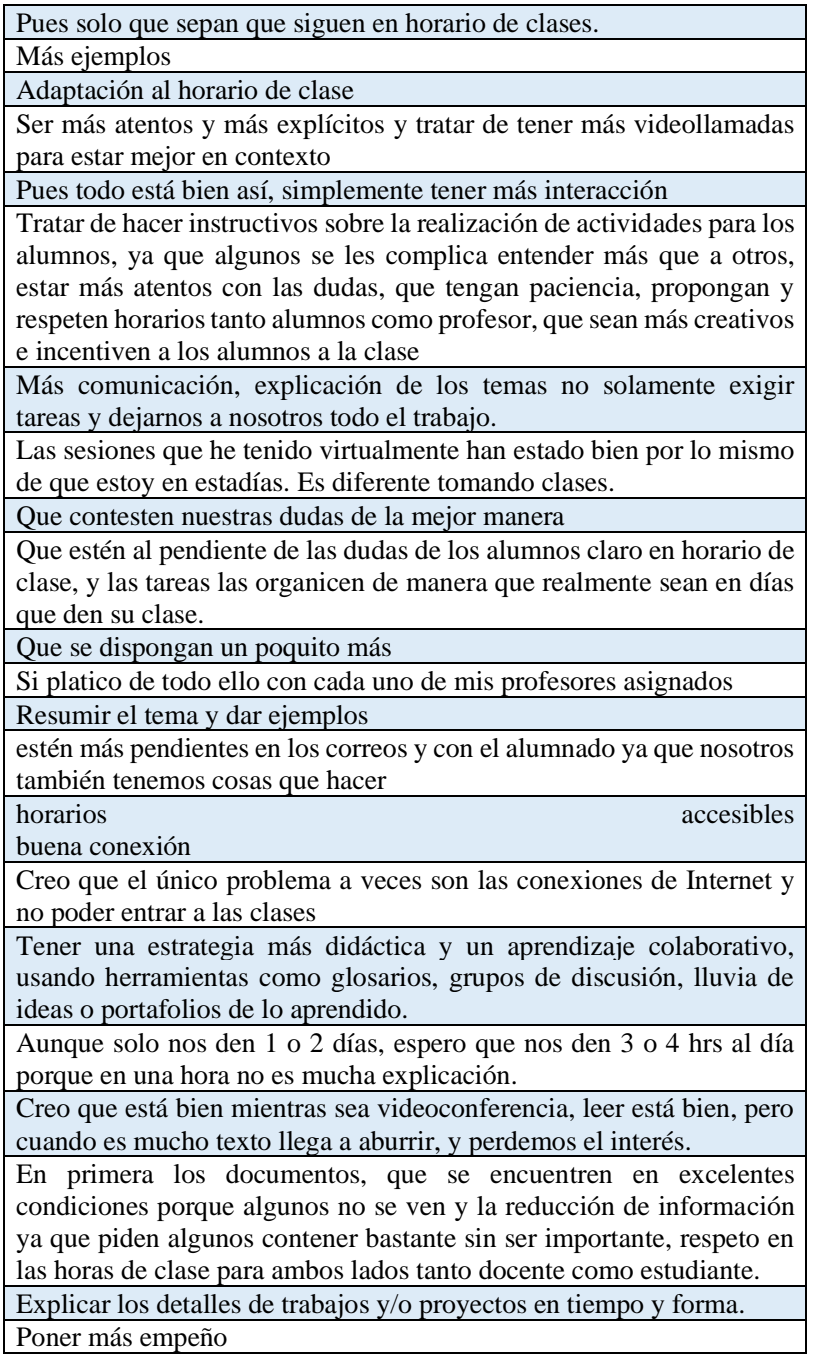

Gráfico 8 Expresa tus sugerencias para tus profesores en cuanto a la dinámica de clases virtuales y la mejora de estas
El punto focal de esta modalidad: la función y desempeño docente. Sobre ello el análisis llevaría un artículo especial, sin embargo, es clara la demanda estudiantil y es que el docente se adecúe al modelo virtual, prevea las actividades y analice los resultados previos de las mismas. Destaca la necesidad individual de atención, en el sentido de obtener un aprendizaje significativo, lo impersonal que se vuelve en el momento que es a través del monitor.

Resulta interesante que, en este caso particular, el alumnado provee de sugerencias y atañe el respeto por los tiempos asignados. Se reconoce como un eslabón importante en la construcción del conocimiento y al docente como guía en la formación. La retroalimentación que brindan los espacios de comunicación de este tipo permite enriquecer las dinámicas, ampliar la visión de las necesidades y los intereses colectivos.

\section{8. ¿Qué consideras que puedes mejorar para aprovechar mucho más las sesiones en modalidad virtual?}

\begin{tabular}{|c|}
\hline Pues cambiar mi mentalidad y tener una actitud más positiva. \\
\hline No sé hay mayor facilidad de distracción \\
\hline No sé \\
\hline Nada \\
\hline Teniendo más sesiones \\
\hline La creatividad \\
\hline La atención de los profesores \\
\hline $\begin{array}{l}\text { Es que es difícil aprovecharlo al } 100 \% \text {. Todos aprendemos de } \\
\text { manera distinta, la mía es más visual y verbal por lo que no me } \\
\text { ayuda a nada ver al maestro en una pantalla y estar horas } \\
\text { sentada en una silla sin interacciones. }\end{array}$ \\
\hline Entender más las clases y utilizar mejor el sistema virtual \\
\hline $\begin{array}{l}\text { Videos de los profesores explicando el tema para comprender } \\
\text { mejor. }\end{array}$ \\
\hline Estar más enfocado \\
\hline $\begin{array}{l}\text { Tratar de aprovechar más los cursos, pláticas y demás que se } \\
\text { están Impartiendo }\end{array}$ \\
\hline Tomar notas de dudas que no comprenda del tema \\
\hline ninguna \\
\hline adentrarme a los temas vistos \\
\hline Pues asistir a la universidad \\
\hline $\begin{array}{l}\text { Mi motivación y participación, para tener más concentración } \\
\text { en mis clases. }\end{array}$ \\
\hline El cumplimiento y la puntualidad de mi parte. \\
\hline El tiempo que le dedico \\
\hline $\begin{array}{l}\text { Sería la presencia de las clases, respetar los horarios, pero } \\
\text { también no hay que olvidar que existen muchos factores que } \\
\text { pueden arruinar las clases virtuales, por ejemplo; el internet } \\
\text { que en muchos casos se va o se roban los cables, otra es la } \\
\text { computadora, en el celular no es malo escuchar las clases, pero } \\
\text { hay que recordar que muchos no poseen o tienen problemas } \\
\text { con sus máquinas }\end{array}$ \\
\hline $\begin{array}{l}\text { Hacer notas apropiadas de lo más importante o de datos } \\
\text { interesantes extras. }\end{array}$ \\
\hline Más constancia \\
\hline
\end{tabular}

Gráfico 9 ¿Qué consideras que puedes mejorar para aprovechar mucho más las sesiones en modalidad virtual? 
En este último sector, se exponen los dos ámbitos de visión del alumnado: el que sí reconoce y pretende un mayor esfuerzo para la mejora continua y el que se siente estancado, sin nada más que poder dar y en una inminente necesidad de interacción física con la comunidad escolar. El manejo de las emociones y administración del tiempo, vuelven a presentarse, como un requerimiento imperioso para la formación integral del estudiantado.

\section{Conclusiones}

Ha sido sin duda, la pandemia por COVID-19 una época compleja para el entorno educativo. De la noche a la mañana los jóvenes universitarios han tenido que lidiar con una nueva forma de aprendizaje y realización de actividades $\mathrm{y}$, sobre todo, con una forma inédita de convivencia académica y social que incide en su capacidad para aprender, debido al cambio sustancial en la modificación del proceso de enseñanza-aprendizaje. (Olivera, 2020).

Así lo demuestran este grupo de estudiantes en sus ávidas respuestas. La adaptación al cambio, la necesidad de interactuar físicamente y dentro de las instalaciones de la Universidad, de lo que para ellos se convierte en un segundo hogar, espacio de aprendizaje y desarrollo integral; son imperantes.

El entorno familiar ha sido modificado, el esfuerzo continuo por mejorar la comunicación y la convivencia generan un estrago de presión o estrés que el joven por sí solo no sabe resolver. Esto merece especial atención del cuidado de la salud emocional, implementar un programa de apoyo a través del departamento psicopedagógico y que coadyuve en la mejora del proceso del aprendizaje. En cuanto a la salud física es motivar al alumnado y/o apoyarle en la administración de su tiempo para que participe de actividades físicas organizadas por el departamento de deporte y cultura de la misma institución y que sean transmitidas por algún canal de interactivo al que todas y todos tengan acceso.

Es necesario también la evaluación de los recursos digitales de la comunidad estudiantil y en apoyo con sectores gubernamentales, asegurar el acceso igualitario a internet, asegurando una conectividad efectiva. Como se mencionó en varias respuestas, la calidad de señal afecta directamente al modelo y es causa de ausencias y desánimos.
En el grupo, todos y todas cuentan con equipos digitales y actualmente hay programas del Estado que apoyan la adquisición de alguno; sin embargo, de nada sirve si la conectividad no es eficiente.

Meritorio ha sido el trabajo docente, la suma de esfuerzos permitió la continuidad en casa de la educación, no obstante, es obvia la necesidad de capacitación para la integración de tecnologías al proceso enseñanza-aprendizaje. Muchos ha sido los ejemplos de profesores y profesoras que a pesar del esfuerzo fueron rebasados por la modalidad virtual, que la capacidad de transmitir el conocimiento a través de un monitor se ha vuelto lenta y por supuesto, se refleja en el interés y seguimiento del alumnado. Brindar herramientas, capacitación constante, líneas de comunicación efectiva y retroalimentación objetiva en el desarrollo docente, es vital para el subsistir del modelo virtual.

Aunque este es sólo un grupo muestra mínimo. de lo que la comunidad universitaria representa, el sentir es generalizado y nos da la oportunidad de repensar el cómo se ha respondido a esta enseñanza virtual y qué áreas de oportunidad se presentan ante el reto que inunda a todo el sector educativo. Ha habido grandes adaptaciones, se han atendido los principales focos rojos, pero aún queda una brecha que llenar y lo importante es comprender que el conocimiento se construye en conjunto: autoridades, alumnado, docentes y familia conforman cada uno un eslabón fundamental en la formación integral de una ciudadanía responsable y colaborativa.

\section{Referencias}

INEGI De 2015-2018: Encuesta Nacional sobre Disponibilidad y Uso de TIC en Hogares, ENDUTIH.

Ivonne Ramírez, Carla Jaliri, Bernarda Méndez Roca, Ingrid Orlandini. (2020). ¿Cómo perciben los universitarios la educación virtual en tiempos de COVID-19?. 25/11/2020, de ASEFIE Sitio web: http://formacionib.org/noticias/?Comoperciben-los-universitarios-la-educacionvirtual-en-tiempos-de-COVID-19 
José Antonio Quinteiro Goris. (2020).

IMPACTO DE LA CRISIS DEL

CORONAVIRUS COVID-19 EN LAS IES: RESPUESTAS A LA EMERGENCIA Y TRANSFORMACIÓN DIGITAL DE LA EDUCACIÓN SUPERIOR. 26/11/2020, de IESALC Sitio web: https://www.iesalc.unesco.org/2020/07/17/dialo go-sobre-el-impacto-de-la-crisis-delcoronavirus-covid-19-en-las-instituciones-deeducacion-superior-respuestas-a-la-emergenciay-transformacion-digital-de-la-educacionsuperior/

Olivera, F. (3 de junio de 2020). Percepción de estudiantes universitarios sobre la enseñanza en línea durante la pandemia de covid-19: algunos apuntes. Notas de coyuntura del crim No. 25, México, crim-unam, 6 pp.

Pierini, N. (7 de abril de 2020). El coronavirus: la oportunidad más perfecta para la educación a distancia. Students for Liberty. A Freer Future. Recuperado de https:// studentsforliberty.org/eslibertad/blog/elcoronavirus-la-oportunidad-masperfecta-parala-educacion-a-distancia/

Saavedra, J. (2020). covid-19 y educación: algunos desafíos y oportunidades. Education for global development. A blog about the power of investing in people. World Bank. Recuperado de https://blogs.worldbank.org/es/education/educat ional-challengesand-opportunities-covid-19pandemic 